\title{
RE-SHAPING MALLOTUS [PART 1]: EXPANDED CIRCUMSCRIPTION AND REVISION OF THE GENUS CORDEMOYA (EUPHORBIACEAE)
}

\author{
S.E.C. SIERRA, M. APARICIO, K.K.M. KULJU, Ž. FIŠER, \\ P.C. VAN WELZEN \& R.W.J.M. VAN DER HAM \\ Nationaal Herbarium Nederland, Universiteit Leiden branch, \\ P.O. Box 9514, 2300 RA Leiden, The Netherlands
}

\begin{abstract}
SUMMARY
A recent phylogenetic study based on DNA sequence data detected a well-supported clade of a number of Mallotus species together with the genus Cordemoya. This clade is distinct from the large Mallotus s.s. clade. In this paper, Cordemoya (formerly monotypic with $C$. integrifolia from the Mascarene Islands) is expanded with sixteen additional species previously assigned to Mallotus. Taxa transferred to Cordemoya are: Mallotus sections Hancea and Oliganthae from Asia and M. baillonianus, M. capuronii, and M. spinulosus from Madagascar. The genus Cordemoya can be distinguished from Mallotus s.s. by the presence of pollen with areolate ornamentation with scabrae (perforate/microreticulate ornamentation with scabrae in the sample of Mallotus s.s. studied up to now) and of capitate glandular hairs with multicellular stalks and sessile peltate-stellate hairs with a central cell (spherical to disc-shaped multicellular glandular hairs in Mallotus). In the new circumscription of Cordemoya two subgenera are recognized: Cordemoya from Madagascar and Mascarenes and Diplochlamys from Asia. The latter is divided into two sections: Diplochlamys (former sect. Hancea), and Oliganthae. A taxonomic revision of part of these taxa is provided (excl. section Diplochlamys); descriptions, distribution maps, habit drawings and a key are included.
\end{abstract}

Key words: Euphorbiaceae, Cordemoya, Deuteromallotus, Mallotus, Malesia, Madagascar, Mauritius, Réunion.

\section{INTRODUCTION}

In the last classification of Mallotus Lour. by Airy Shaw (1968), the genus was subdivided into eight sections based on few morphological characters. However, two phylogenetic studies have recently contributed to the knowledge of the boundaries of the genus Mallotus and its relationships with related genera, namely with the members of subtribe Rottlerinae Meisn. (Webster, 1994) and the genus Macaranga Thouars. An analysis of morphological data (Slik \& Van Welzen, 2001b) indicated that the Asian sections Hancea (Seem.) Pax \& K. Hoffm. (12 spp.) and Oliganthae Airy Shaw (monotypic) might not belong to the same clade as the other Mallotus sections and the genus Macaranga. A study of DNA sequence data with a more extensive taxon sampling (Kulju et al., in prep.) confirmed this result, grouping these two sections together with three Mallotus species (= Deuteromallotus) endemic to Madagascar (M. baillonianus Müll.Arg., M. capuronii (Léandri) McPherson, and M. spinulosus McPherson), and 


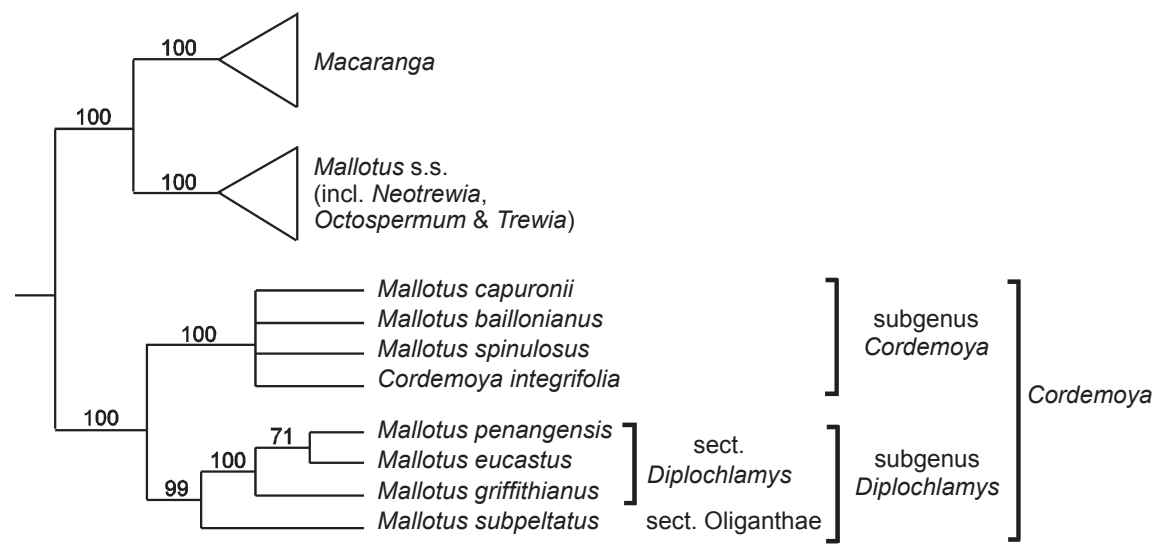

Fig. 1. A tree summarizing the phylogenetic relationships of Macaranga, Mallotus and related small genera (adapted from Fig. $2 \& 3$ in Kulju et al., in prep.). Maximum parsimony bootstrap values are given above branches. On the right the new circumscription and classification of the genus Cordemoya.

monotypic Cordemoya from Mauritius and Réunion. This clade is in a basal position compared to the Mallotus s.s. clade (with monotypic Neotrewia Pax \& K. Hoffm., Octospermum Airy Shaw, and Trewia L.) and the Macaranga clade (Fig. 1). All these three clades have strong bootstrap support.

Consequently, the circumscription of Mallotus has to be changed to make the genus monophyletic. The inclusion of Neotrewia, Octospermum, and Trewia is discussed separately (Kulju \& Van Welzen, in prep.). In this paper, Mallotus sections Hancea and Oliganthae, and three endemic Madagascan Mallotus species are transferred to the genus Cordemoya, which now consists of seventeen species: C. acuminata (Baill.) Baill., C. capuronii (Léandri) S.E.C. Sierra, Kulju \& Welzen, C. cordatifolia (Slik) S.E.C. Sierra, Kulju \& Welzen, C. eucausta (Airy Shaw) S.E.C. Sierra, Kulju \& Welzen, C. grandistipularis (Slik) S.E.C. Sierra, Kulju \& Welzen, C. griffithiana (Müll.Arg.) S.E.C. Sierra, Kulju \& Welzen, C. hirsuta (Elmer) S.E.C. Sierra, Kulju \& Welzen, C. hookeriana (Seem.) S.E.C. Sierra, Kulju \& Welzen, C. integrifolia (Willd.) Baill., C. kingii (Hook.f.) S.E.C. Sierra, Kulju \& Welzen, C. longistyla (Merr.) S.E.C. Sierra, Kulju \& Welzen, C. papuana (J.J. Sm.) S.E.C. Sierra, Kulju \& Welzen, C. penangensis (Müll.Arg.) S.E.C. Sierra, Kulju \& Welzen, C. spinulosa (McPherson) S.E.C. Sierra, Kulju \& Welzen, C. stipularis (Meijer ex Airy Shaw) S.E.C. Sierra, Kulju \& Welzen, C. subpeltata (Blume) M. Aparicio, and C. wenzeliana (Slik) S.E.C. Sierra, Kulju \& Welzen. The morphological characters typical for Cordemoya and its morphological differences with Mallotus s.s. are discussed. An infrageneric classification with two subgenera and two sections is also proposed. Finally, a revision of Cordemoya with a key, descriptions, distribution maps and drawings is given (excluding section Diplochlamys). 


\section{MORPHOLOGICAL AND ANATOMICAL CHARACTERS OF MALLOTUS AND CORDEMOYA}

The molecular support for the new circumscription of Mallotus and Cordemoya is strong; however, there are only few uniquely distinguishing morphological characters separating them. One distinct character is in the pollen ornamentation (Fig. 2): the genus Cordemoya has pollen with areolate ornamentation with scabrae instead of perforate/ microreticulate ornamentation with scabrae as found in Mallotus s.s. [N.B. the genus has largely been sampled so far, but not completely, therefore other kinds of ornamentation could be present, including areolate]. Leaf anatomical studies have revealed the presence of capitate glandular hairs with multicellular stalks (in subg. Diplochlamys and $C$. capuronii, Fig. $3 \mathrm{c}$ ) and of sessile peltate-stellate hairs with a central cell (in subg. Cordemoya, Fig. 3a, b), instead of spherical to disc-shaped multicellular glandular hairs found in Mallotus (Fig. 3d-f; Fišer et al., in prep.). Macromorphological differences also exist, but many of them overlap, often because of a few deviating Mallotus s.s. species. The main typical characters of Cordemoya are: extrafloral nectaries on the upper leaf surface absent or inconspicuous when present (always present and conspicuous in Mallotus); staminate inflorescences with 1-3 flowers per node (1-15 in Mallotus); pistillate inflorescences racemes or developing one terminal flower (racemes, panicles, spikes or umbels in Mallotus, with the exception of Trewia nudiflora L. (to be transferred to Mallotus together with T. polycarpa Benth., Kulju, in prep.), which sometimes develops only one terminal flower); stigmas erect and long, 5-22 mm (mostly reflexed and short, 0.7-11 mm in Mallotus, with the exception of M. khasianus Hook.f., 5-14 mm, T. polycarpa 10-12 mm, and T. nudiflora, 12-24 mm); and spiny fruits (spiny or not in Mallotus).

\section{INFRAGENERIC CLASSIFICATION}

The rank of subgenus was assigned to the two strongly supported and geographically distinct Cordemoya clades present in the phylogeny of Kulju et al. (in prep.; Fig. 1): subgenus Cordemoya consisting of four species from Madagascar and Mascarenes and subgenus Diplochlamys consisting of the Asian species. Furthermore, the subgenus Diplochlamys was subdivided into sections Diplochlamys and Oliganthae, corresponding to the former sections Hancea and Oliganthae in Mallotus s.l., respectively (Fig. 1).

Morphologically, these three taxa can be distinguished by leaf arrangement. In subg. Cordemoya the leaves are predominantly opposite and similar in shape, but one only slightly smaller than the other (sometimes the leaves are alternate). Additionally, it is the only subgenus which has sessile peltate-stellate hairs with a central cell. The members of section Diplochlamys also have opposite leaves, but the smaller leaf of each pair is reduced and dissimilar, resembling a stipule. Section Oliganthae always has alternate leaves. Sections Diplochlamys and Oliganthae have capitate glandular hairs with multicellular stalks, which are also present in C. capuronii of subgenus Cordemoya. Further characters separating section Diplochlamys from section Oliganthae are branches with conspicuously swollen nodes (slender in Oliganthae), interpetiolar stipules (axillary in Oliganthae), leaf blade not peltate (subpeltate in Oliganthae), axillary inflorescences (ramiflorous and axillary in Oliganthae), and fruits opening septicidally-loculicidally (loculicidally-septicidally in Oliganthae). 

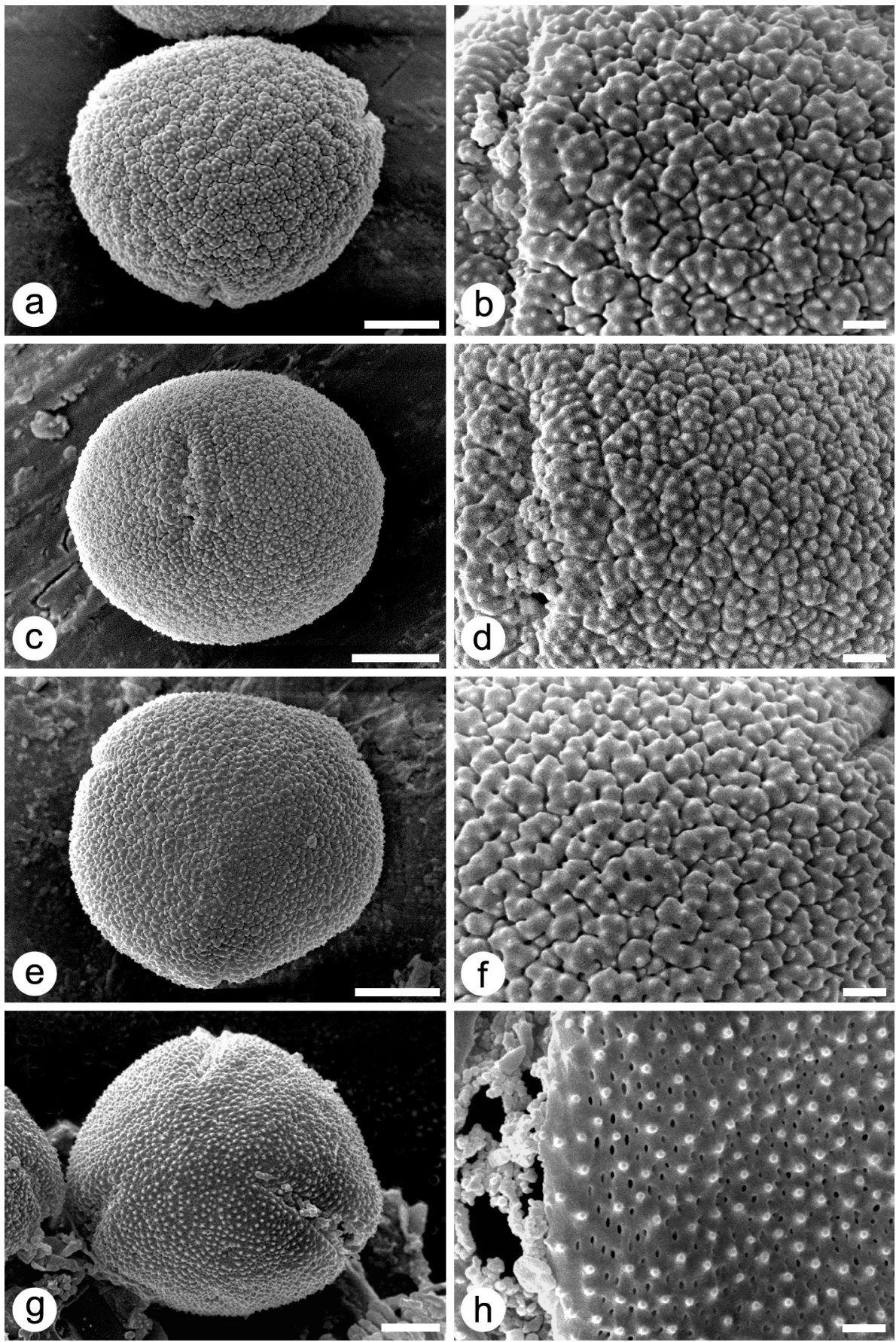

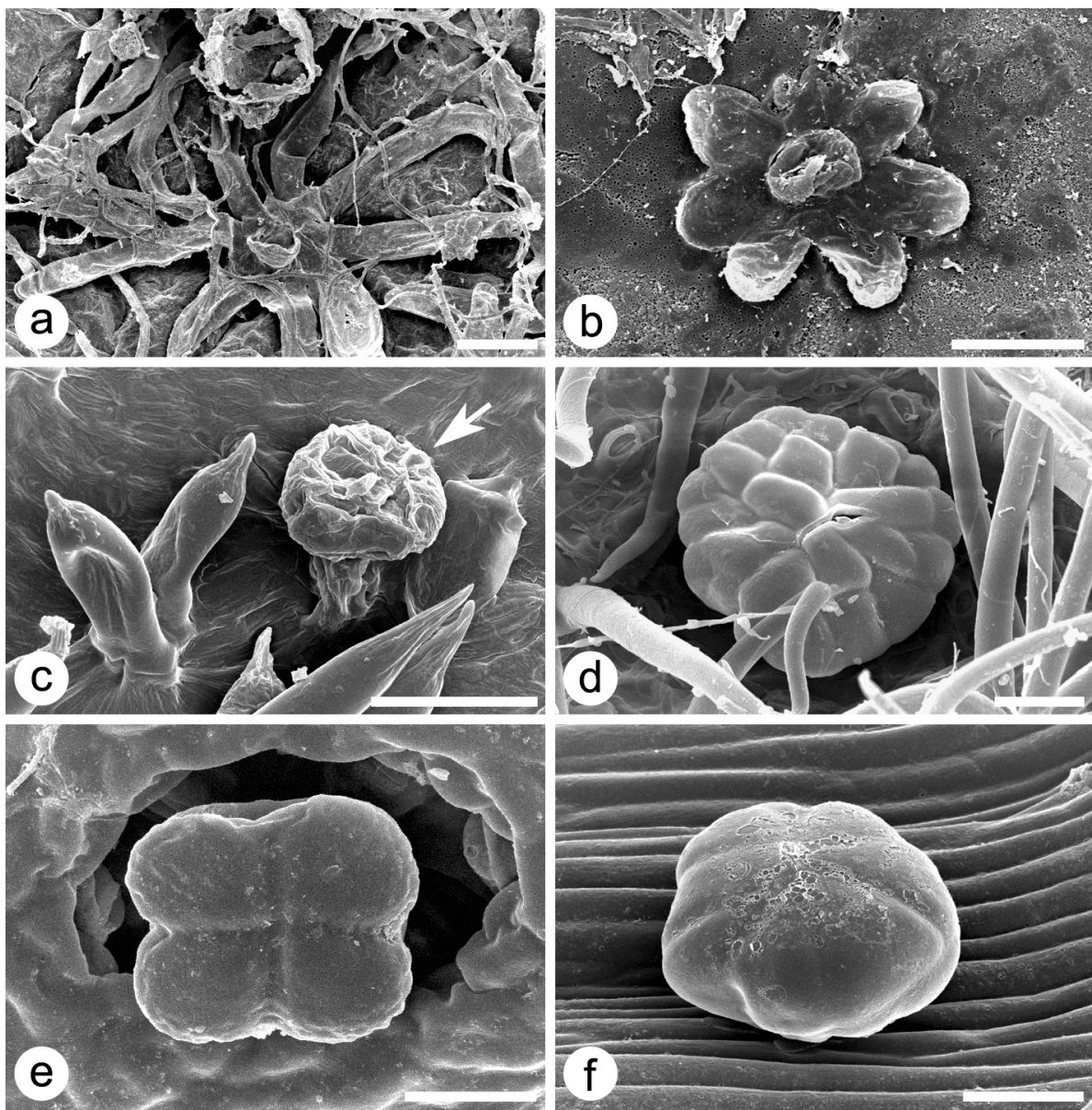

Fig. 3. Cordemoya and Mallotus hairs and glandular hairs. - a. Cordemoya capuronii (Léandri) S.E.C. Sierra, Kulju \& Welzen. Sessile peltate-stellate hair with a central cell. - b. Cordemoya spinulosa (McPherson) S.E.C. Sierra, Kulju \& Welzen. Sessile peltate-stellate hair with a central cell. - c. Cordemoya subpeltata (Blume) M. Aparicio. Capitate glandular hair with multicellular stalks. - d. Mallotus repandus (Rottler) Müll. Arg. Spherical multicellular glandular hair. - e. Mallotus resinosus (Blanco) Merr. Disk-shaped multicellular glandular hairs. - f. Mallotus lackeyi Elmer. Spherical multicellular glandular hair (a: Messmer \& Andriatsiferana 698, G; b: Totozafy Be 520, WAG; c: Prawiroatmodjo 361, L; d: Stocks s.n., L; e: Craven \& Schodde 999, L; f: Ambriansyah \& Abrainsyah B805, L). - Scale bars: $\mathrm{a}-\mathrm{f}=25 \mu \mathrm{m}$.

Fig. 2. Cordemoya and Mallotus pollen micrographs. - a, b: Cordemoya capuronii (Léandri) S.E.C. Sierra, Kulju \& Welzen. a. Polar view of 3-colporate grain; b. detail of ornamentation. c, d: Cordemoya subpeltata (Blume) M. Aparicio. c. Equatorial view; d. detail of ornamentation. e, f: Cordemoya papuana (J.J. Sm.) S.E.C. Sierra, Kulju \& Welzen. e. Polar view of 3-colporate grain; f. detail of ornamentation. - g, h: Mallotus hymenophyllus Airy Shaw. g. Polar view of 3-colporate grain; h. detail of ornamentation (a, b: Labat \& Andrianjafy 3399, G; c, d: Maxwell 86-133, L; e, f: Lam 7805, L; g, h: Larsen et al. 31247, L). - Scale bars: a, c, e, g $=5 \mu \mathrm{m}$; b, d, f, h $=1 \mu \mathrm{m}$. 


\section{TAXONOMIC HISTORY}

The first generic name for the basal (Cordemoya) clade is Boutonia Bojer (1837), but this name was invalidly described, the only descriptive 'character' casually mentioned is 'shrub'. The validation in 1846 by Bojer created a later homonym, because De Candolle (1838) had used Boutonia for an Acanthaceae.

The earliest legitimate generic name is Cordemoya Baill. (1861), which therefore is adopted here. It was proposed for Ricinus integrifolius Willd. from Mauritius. Baillon (1861) transferred the latter species to his new genus Cordemoya and added C. acuminata Baill. from Madagascar. Several years later Baillon (1891a) moved these two species to Echinus Lour. as sect. Cordemoya.

Müller Argoviensis (1865) transferred them to Mallotus sect. Cordemoya Müll. Arg., in which he also included M. penangensis Müll.Arg., a Southeast Asian species. This concept already anticipated the recent phylogenetic results (Kulju et al., in prep.) by grouping these geographically distinct species together. Airy Shaw (1968) placed M. penangensis in sect. Hancea and neither discussed the African species nor Cordemoya in literature (though he had his own ideas about the circumscription of Cordemoya, which was mainly based on fruit, flower and leaf morphology; see Coode, 1982).

Diplochlamys Müll.Arg. (1864) was monotypic (D. griffithianus Müll.Arg.). Bentham (1880) reduced it to Mallotus, while Pax (1890) regarded it as a section of Mallotus and added M. subpeltatus (Blume) Müll.Arg. However, Airy Shaw (1968) included the first species in sect. Hancea and the second in his new sect. Oliganthae. The section name Hancea dates from 1914 (Pax \& Hoffmann), is younger than Diplochlamys and should therefore be regarded as a synonym. Most systematists either missed the fact that the name Diplochlamys was used on sectional level (e.g., Airy Shaw, 1968) or they failed to notice that Diplochlamys is an older name (e.g., Slik \& Van Welzen, 2001a).

Deuteromallotus Pax \& K. Hoffm. (1914) was described to accommodate Boutonia acuminata Baill. (1858). The classification of this species has varied much through time. Baillon (1861) placed it in Cordemoya, Müller Argoviensis (1865) put it in Mallotus sect. Cordemoya and, because of the already existing M. acuminatus (Blume) Müll.Arg. (1865), he renamed the species M. baillonianus Müll.Arg. Baillon (1891a) moved M. baillonianus to Echinus Lour. Finally, McPherson (1995) transferred it to Mallotus. Léandri (1957) added the compatriot M. capuronii to Deuteromallotus, which McPherson (1995) also placed in Mallotus.

\section{CORDEMOYA Baill.}

Cordemoya Baill. (1861) 255. - Boutonia Bojer (1837) 282, nom. nud.; (1846) 151, non DC. (1838). - Mallotus Lour. sect. Cordemoya (Baill.) Müll.Arg. (1865) 186. - Echinus Lour. sect. Cordemoya (Baill.) Baill. (1891a) 977. - Type: Boutonia mascareinensis Bojer [= Cordemoya integrifolia (Willd.) Baill.].

Hancea Seem. (1857) 409. - Mallotus Lour. sect. Hancea (Seem.) Pax \& K. Hoffm. (1914) 199. - Type: Hancea hookeriana Seem. [= Cordemoya hookeriana (Seem.) S.E.C. Sierra, Kulju \& Welzen].

Diplochlamys Müll. Arg. (1864) 539; (1866) 1023. - Mallotus Lour. sect. Diplochlamys (Müll.Arg.) Baill. ex T. Durand (1888) 370; Müll.Arg. ex Pax (1890) 55, isonym. - Type: D. griffithianus Müll.Arg. [= Cordemoya griffithiana (Müll.Arg.) S.E.C. Sierra, Kulju \& Welzen]. 
Deuteromallotus Pax \& K. Hoffm. (1914) 212. - Type: Deuteromallotus acuminatus (Baill.) Pax $\&$ K. Hoffm. [= Cordemoya acuminata (Baill.) Baill.].

Mallotus Lour. sect. Oliganthae Airy Shaw (1968) 389. - Type: Mallotus subpeltatus Blume [= Cordemoya subpeltata (Blume) M. Aparicio].

Shrubs to big trees, monoecious and/or dioecious. Branches with slender or conspicuously swollen nodes. Indumentum composed of simple hairs and sometimes capitate glandular hairs with multicellular stalks (subg. Diplochlamys and C. capuronii), or sessile peltate-stellate hairs with a central cell (subg. Cordemoya). Resin sometimes present (C. integrifolia and C. spinulosa). Stipules axillary or interpetiolar. Leaves simple, alternate or opposite (strongly unequal or not), (sub)coriaceous to chartaceous, adaxial extrafloral nectaries absent or when present inconspicuous, midrib, nerves and veins prominent, venation triplinerved, (rarely palminerved) or pinnate, veins scalariform, veinlets reticulate. Inflorescences ramiflorous, axillary, or terminal, unisexual to bisexual, with one bract per node; staminate ones with 1-3 flowers per bract, bracts persistent, bracteoles absent; pistillate ones with 1 flower per bract, bracts persistent to caducous, bracteoles rarely present. Flowers actinomorphic, not exceeding $1 \mathrm{~cm}$ diam.; sepals persistent; petals and disc absent. Staminate flowers: sepals 2-4, valvate, free to basally connate; stamens: thecae 2, parallel, opening extrorsely and lengthwise; pollen ornamentation areolate with scabrae; pistillode absent. Pistillate flowers: sepals ( 3 or) 4-6 (or 7), valvate or imbricate, free; staminodes rarely present; ovary with 1 ovule per locule; style short, stigmas erect, inner surface densely covered with short papillae, outer surface hairy. Fruits dehiscent capsules, opening loculicidally-septicidally or septicidally-loculicidally, spiny, spines sometimes with terminal glands. Seeds (more or less) globose, somewhat trigonous in transverse section; caruncle or aril absent.

Distribution - Madagascar, Mauritius, Réunion, from South China throughout Southeast Asia and Malesia to New Guinea.

\section{KEY TO THE SPECIES}

1a. Leaves alternate or opposite with one leaf of a pair similar in shape and only slightly smaller than the other. Stipules axillary. . . . . . . . . . . . . 2

b. Leaves opposite with one leaf of a pair stipuliform. Stipules interpetiolar. . . . . . Sect. Diplochlamys (see Slik \& Van Welzen, 2001a, for key, descriptions, distributions, and figures)

2a. Leaves opposite or sometimes alternate, base not peltate, pinnately nerved. Stipules linear-triangular 3-20 by $0.2-1 \mathrm{~mm}$, margin entire. Inflorescences axillary or terminal. Staminate inflorescences $2-10 \mathrm{~cm}$ long. - Madagascar, Mauritius and

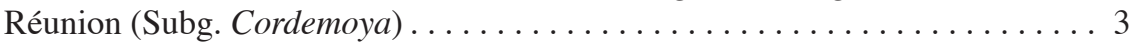

b. Leaves alternate, base subpeltate up to $2 \mathrm{~mm}$, triplinerved. Stipules narrowly triangular 5-7 by 1.8-2 mm, margin serrate. Inflorescences ramiflorous or axillary. Staminate inflorescences $0.5-0.8 \mathrm{~cm}$ long. - Burma to Thailand, Peninsular Malaysia, Sumatra, Java, and Borneo (Sect. Oliganthae)... . 17. C. subpeltata

3a. Leaves ovate. Stipules 6.5-20 mm long. Stamens 200-250. - Mauritius and

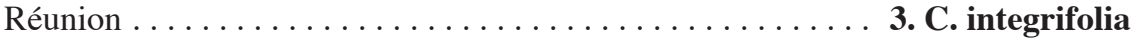

b. Leaves ovate, elliptic or obovate. Stipules 2-12 mm long. Stamens 70-150. Madagascar ........................ 4 
4a. Leaves obovate, apex emarginate, obtuse, rounded, to rarely acute or shortly acu-

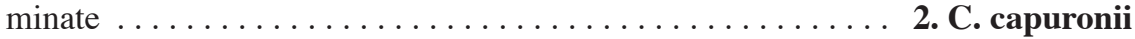

b. Leaves ovate to elliptic, apex acuminate to caudate $\ldots \ldots \ldots \ldots \ldots \ldots$

5a. Young branches, petioles, leaf venation on lower surface and inflorescences densely to sparsely hairy with long and soft hairs. Stipules persistent to caducous, 5-12 mm. Leaf apex caudate. Fruit $15-25$ by $30-35$ mm, 2-locular, spines $80-100$. .

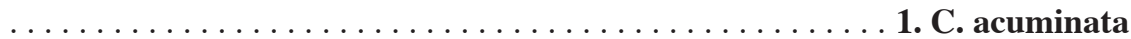

b. Young branches, petioles, leaf venation on lower surface and inflorescences (sub) glabrous. Stipules early caducous, $2-5 \mathrm{~mm}$. Leaf apex acuminate. Fruit 5-6.5 by $8-9 \mathrm{~mm}, 2-$ or 3 -locular, spines $4-6 \ldots \ldots \ldots \ldots \ldots \ldots$ 4. C. spinulosa

\section{Subgenus Cordemoya}

Cordemoya Baill. (1861) 255. - Boutonia Bojer (1837) 282, nom. nud.; (1846) 151, non DC. (1838). - Mallotus Lour. sect. Cordemoya (Baill.) Müll.Arg. (1865) 186. - Mallotus Lour. sect. Boutonia Benth. (1880) 319. - Echinus Lour. sect. Cordemoya (Baill.) Baill. (1891a) 977. - Type: Boutonia mascareinensis Bojer [= Cordemoya integrifolia (Willd.) Baill.].

Deuteromallotus Pax \& K. Hoffm. (1914) 212. - Type: Deuteromallotus acuminatus (Baill.) Pax $\&$ K. Hoffm. [= Cordemoya acuminata (Baill.) Baill.].

Branches with slender nodes. Indumentum composed of simple and sessile peltatestellate hairs with a central cell and capitate glandular hairs with multicellular stalks (C. capuronii). Stipules axillary, margin entire. Leaves opposite (in C. capuronii, C. integrifolia, and $C$. spinulosa sometimes alternate), unequal in size; blade not peltate, (sub)coriaceous, margin entire to crenate, extrafloral nectaries present or absent, venation pinnate with the lowest nerves undifferentiated and submarginal. Inflorescences axillary or terminal. Staminate inflorescences racemes (sometimes with 1-3 side branches), with 1-3 flowers per bract. Male flowers: sepals 2-4, valvate, basally connate; connective not widened. Pistillate inflorescences racemes and/or developing one flower terminally. Pistillate flowers: sepals 4-6; ovary 2- or 3-locular. Fruits opening septicidally-loculicidally, inside glabrous, spines without terminal glands.

Distribution - Madagascar, Mauritius, and Réunion.

\section{Cordemoya acuminata (Baill.) Baill. - Fig. 4a-c; Map 1}

Cordemoya acuminata (Baill.) Baill. (1861) 255. - Boutonia acuminata Baill. (1858) 401. - Mallotus baillonianus Müll.Arg. (1865) 186, non M. acuminatus (Blume) Müll.Arg. (1865) 187. - Echinus baillonianus (Müll. Arg.) Baill. (1891a) 977; (1891b) t. 175, nom. superfl. - Deuteromallotus acuminatus (Baill.) Pax \& K. Hoffm. (1914) 212. - Lectotype (McPherson, 1995): Chapelier s.n. (P), Madagascar.

Small tree up to $15 \mathrm{~m}$ high, dbh up to $20 \mathrm{~cm}$, dioecious. Indumentum composed of long and short simple hairs ${ }^{1}$ and sessile peltate-stellate hairs with a central cell. Stipules linear-triangular, $5-12$ by $0.5-1 \mathrm{~mm}$, persistent to caducous, margin entire, apex acute, densely hairy on both sides. Leaves: petiole $10-45$ by $0.5-1 \mathrm{~mm}$, not grooved,

1) Cordemoya acuminata shares with subg. Diplochlamys the presence of simple, short and long $(250-3000 \mu \mathrm{m})$ hairs, primarily found on the leaves (midrib and nerves) and sometimes on the fruits. 


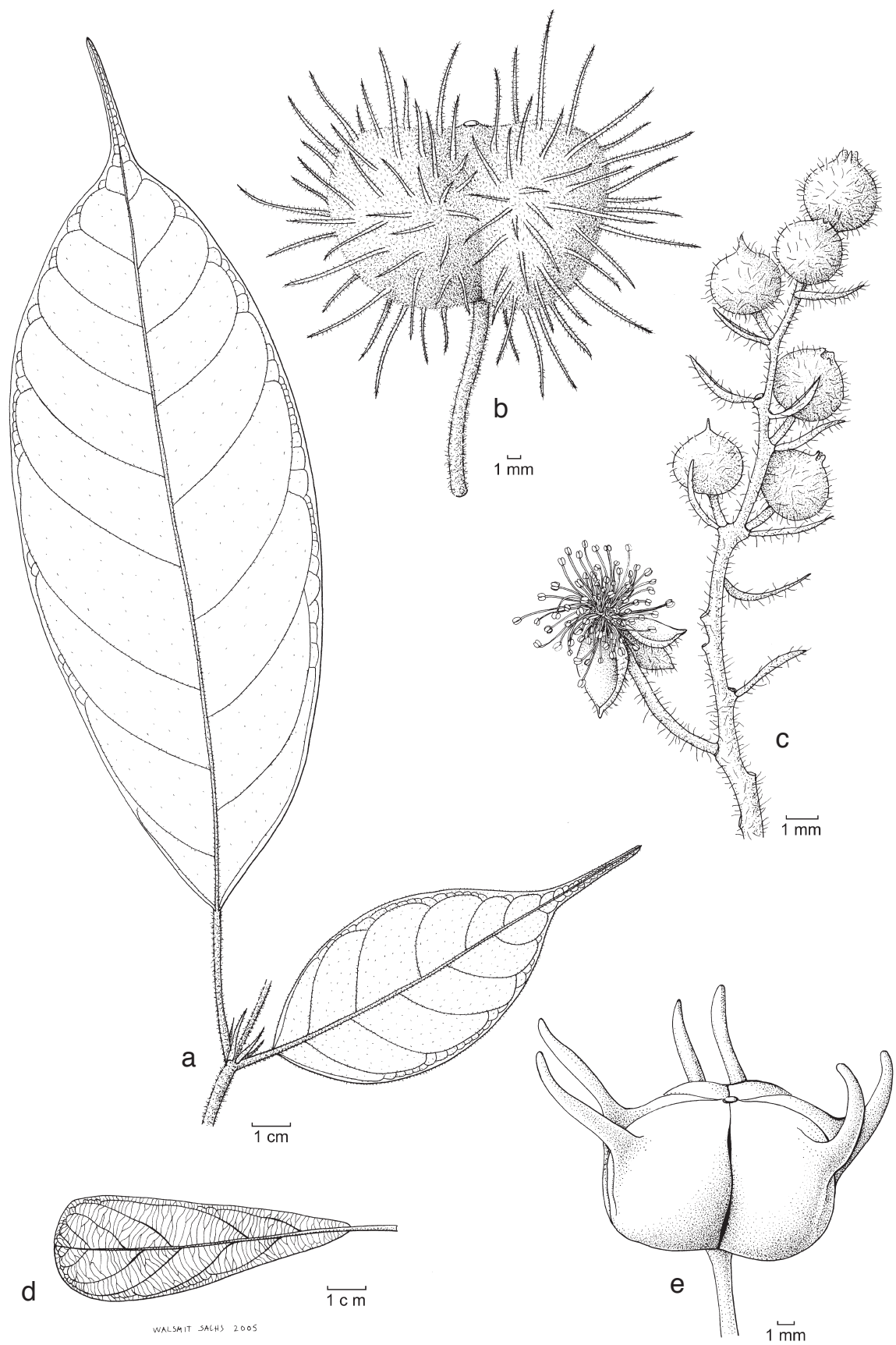

Fig. 4. a-c: Cordemoya acuminata (Baill.) Baill. a. Leaf pair with stipules; b. fruit; c. staminate inflorescence. - d, e: Cordemoya capuronii (Léandri) S.E.C. Sierra, Kulju \& Welzen. d. Leaf; e. fruit (a, c: Aridy \& Moise 184, MO; b: Randrianasolo \& Bernardin 231, G; d: Labat \& Andrianjafy 3399, G; e: Kotozafy 999, MO). 
densely to sparsely hairy; blade elliptic, $8-20$ by $2.8-5 \mathrm{~cm}$, length/width ratio $2.8-4$, subcoriaceous, base not peltate, acute, margin entire to crenate, apex caudate, upper surface sparsely hairy, lower surface densely to sparsely hairy, when young with sessile peltate-stellate hairs with a central cell on both sides, nerves 6-9 per side, looping close to the margin, with hair-tuft domatia. Inflorescences single, unisexual; peduncle 1-3 mm long; bracts linear-triangular, margin entire, apex acute, upper surface densely hairy, lower surface sparsely hairy. Flowers: pedicels densely hairy; sepals: margin entire, apex acute, sparsely hairy outside, subglabrous to glabrous inside. Staminate inflorescences racemes, $4.5-5 \mathrm{~cm}$ long, 1 or 2 flowers per node, nodes per branch 10-17; bracts $4-8$ by $0.3-0.4 \mathrm{~mm}$, bracteoles rarely present, linear-triangular, $0.5-0.8$ by c. $0.1 \mathrm{~mm}$, early caducous. Staminate flowers $4.5-5 \mathrm{~mm}$ diam.; pedicels $2-3 \mathrm{~mm}$ long; sepals 3 or 4 , ovate to obovate, $2-2.5$ by $1.8-2 \mathrm{~mm}$, sparsely hairy outside, glabrous inside; stamens $120-150$, glabrous, filaments $1-3 \mathrm{~mm}$ long, thecae ovoid, $0.2-0.3$ by 0.2-0.25 mm, connective not widened. Pistillate inflorescences developing one flower terminally, $2.5-5 \mathrm{~cm}$ long; sterile bracts $2-5,4-6$ by $0.5-0.8 \mathrm{~mm}$; bracteoles absent. Pistillate flowers 4-5 mm diam.; pedicels c. $1 \mathrm{~mm}$ long; sepals 5 or 6, 5-7 by $0.2-0.25$ $\mathrm{mm}$, valvate; ovary $2.5-3$ by $3-4 \mathrm{~mm}$, 2-locular, densely hairy; style $0.5-1 \mathrm{~mm}$ long; stigma $8-13 \mathrm{~mm}$ long; staminodes absent. Fruits $15-25$ by $30-35 \mathrm{~mm}$, green, outer surface sparsely hairy, spines $80-100$, straight, $5-11$ by $0.5-0.8 \mathrm{~mm}$, sparsely hairy; wall $0.8-1.3 \mathrm{~mm}$ thick; column $6-6.5$ by $8-8.5 \mathrm{~mm}$. Seeds $11.5-12$ by $11.5-12$ by 10-10.5 mm, surface smooth, dull, light brown, striate.

Distribution - Endemic to Madagascar.

Habitat \& Ecology - In evergreen forest. Altitude: sea level up to $1300 \mathrm{~m}$. Flowering and fruiting: October to March.

Vernacular names - Madagascar: Hirondry, kinoronoro, sifidambo.

\section{Cordemoya capuronii (Léandri) S.E.C. Sierra, Kulju \& Welzen, comb. nov. -} Fig. 4d, e; Map 1

Deuteromallotus capuronii Léandri, Bull. Soc. Bot. France 103 (1957, '1956') 605. - Mallotus capuronii (Léandri) McPherson (1995) 170. - Type: SF (MAD) [Capuron] 8713 (holo P; iso MO), Madagascar, Minombo, Amboditavolo.

Small to big trees up to $25 \mathrm{~m}$ high, dbh up to $35 \mathrm{~cm}$, dioecious. Indumentum composed of short simple hairs, sessile peltate-stellate hairs with a central cell and capitate glandular hairs with multicellular stalks. Resin greenish. Stipules linear-triangular, $3-5$ by 0.2-0.4 mm, early caducous, margin entire, apex acute, sparsely hairy on both sides. Leaves: petiole $3-24(-85)$ by $1-1.5(-2.5) \mathrm{mm}$, not grooved, scattered to sparsely hairy; blade obovate, $4.5-7(-22)$ by $1.3-3(-10) \mathrm{cm}$ (see note 1$)$, length/width ratio $2.3-2.5$, coriaceous, base not peltate, acute, margin entire to crenate, apex emarginate to rounded to obtuse (to rarely acute or shortly acuminate), upper surface glabrous, lower surface sparsely hairy, glabrescent, nerves 4-10 per side, looping close to the margin, hair-tuft domatia present to absent. Inflorescences single, unisexual; peduncle 1-3.5 by $1-1.5 \mathrm{~mm}$; bracts linear-triangular, margin entire, apex acute, upper surface densely hairy outside, lower surface densely hairy to subglabrous. Flowers: pedicels glabrous to sparsely hairy; sepals: margin entire, apex acute densely hairy to subglabrous outside, 


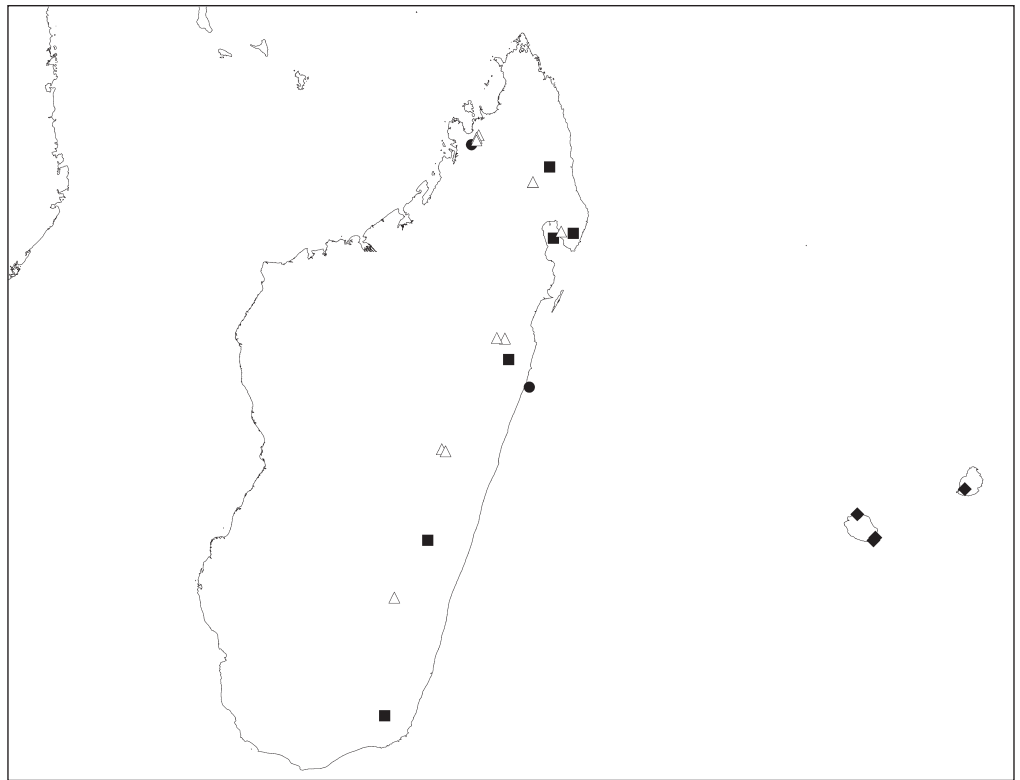

Map 1. Distribution of C. acuminata (Baill.) Baill. (ם), C. capuronii (Léandri) S.E.C. Sierra, Kulju $\&$ Welzen $(\triangle)$, C. integrifolia (Willd.) Baill. ( $\diamond)$, C. spinulosa (McPherson) S.E.C. Sierra, Kulju $\&$ Welzen $(\bullet)$.

glabrous inside. Staminate inflorescences racemes, 2-5.2 cm long, 1 flower per node, nodes per branch $19-30$; bracts $1.5-2(-3)$ by $0.3-0.4(-0.5) \mathrm{mm}$; bracteoles absent. Staminate flowers 3-4 mm diam.; pedicels 2.5-5 mm long; sepals 2 or 3, ovate to obovate, $2.5-4.5$ by $2.5-3 \mathrm{~mm}$, densely hairy outside, glabrous inside; stamens $80-130$, glabrous, filaments $1-3 \mathrm{~mm}$ long, pale light yellow, thecae ovoid, $0.2-0.3$ by $0.2-0.3$ $\mathrm{mm}$, brownish red, connective not widened. Pistillate inflorescences developing one flower terminally, $0.5-1.5 \mathrm{~cm}$ long; bracts $15-30,2.8-3$ by $0.2-0.3 \mathrm{~mm}$; bracteoles $1-3$ by $0.1-0.2 \mathrm{~mm}$, early caducous. Pistillate flowers $2.5-3 \mathrm{~mm}$ diam.; pedicels $1 \mathrm{~mm}$ long; sepals 4 or 5 , linear-triangular, $4-4.5$ by $0.25-0.3 \mathrm{~mm}$, valvate; staminodes absent; ovary $1.8-2$ by $1.8-2 \mathrm{~mm}, 2$ - or 3-locular, densely hairy; style $0.5-1 \mathrm{~mm}$ long; stigma $8-15 \mathrm{~mm}$ long. Fruits $11-13$ by $17-18 \mathrm{~mm}$, green, outer surface subglabrous, spines 4 or 6 (to rarely 8,12 or 20 , see note 2 ), slightly curved, $14-17$ by $0.8-1.2 \mathrm{~mm}$, subglabrous to sparsely hairy; wall c. $0.3-0.5 \mathrm{~mm}$ thick; column $4-5$ by $3.5-4 \mathrm{~mm}$. Seeds $8-9$ by $8-9$ by $8-9 \mathrm{~mm}$, surface smooth, dull, dark brown, striate.

Distribution - Endemic to Madagascar.

Habitat \& Ecology - On edges of humid forest. Altitude 10-1700 m. Flowering and fruiting: September to March.

Vernacular names - Madagascar: Macarenana mavo.

Notes - 1. Andrianjafy et al. 168 and Ratovoson et al. 573 have bigger leaves and male inflorescences compared to the other collections studied.

2. Ravelonarivo \& Rabesonina 653 has 8,12 or 20 fruit spines. 


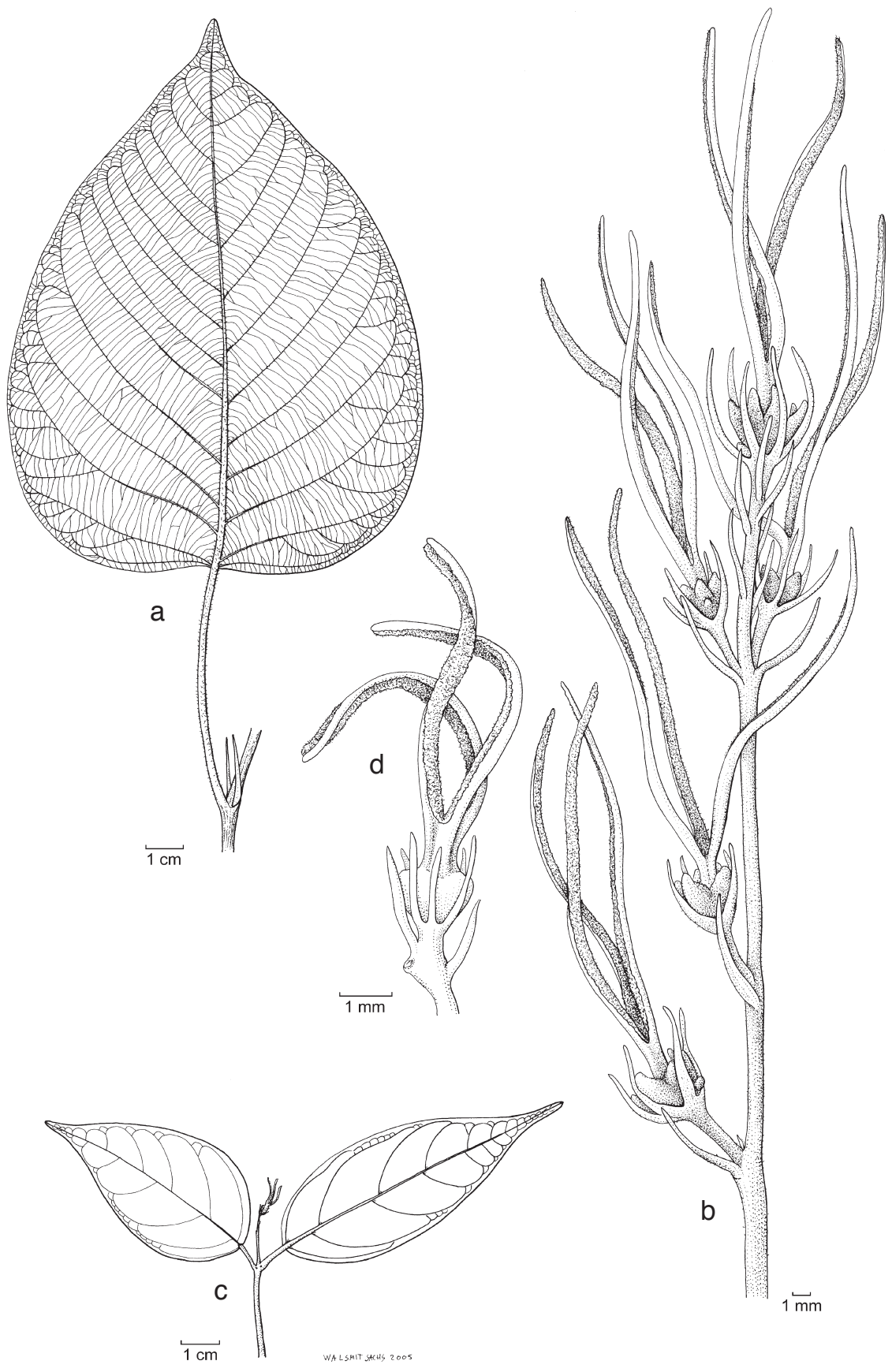

Fig. 5. a, b: Cordemoya integrifolia (Willd.) Baill. a. Leaf with stipules; b. pistillate inflorescence. - c, d: Cordemoya spinulosa (McPherson) S.E.C. Sierra, Kulju \& Welzen. c. Leaf pair with pistillate inflorescence; d. pistillate flower (a: Frappier 278, A; b: Blackburn s.n., A; c, d: Gautier \& Totozafy Be 2910, G). 


\section{Cordemoya integrifolia (Willd.) Baill. - Fig. 5a, b; Map 1}

Cordemoya integrifolia (Willd.) Baill. (1861) 255. - Ricinus integrifolius Willd. (1805) 567. - Boutonia mascariensis Bojer (1837) 282, nom. superfl.; (1846) 151, nom. superfl. - Mallotus integrifolius (Willd.) Müll. Arg. (1865) 186. - Echinus integrifolius (Willd.) Baill. (1891a) 977. - Lectotype (selected by Coode, 1982): Unknown s.n. (Herb. Willd. 17934, B, here limited to the sheet with Radlkofer's notes (IDC microfiche no. 1261)), Mauritius.

Ricinus dioicus Chev. ex Steud. (1841) 459, nom. illegit., non G. Forst. (1786), nec Roxb. (1832). - Voucher: Chapelier s.n. (P?, n.v.).

Ricinus lanceolatus Thouars ex Baill. (1861) 255, nom. nud. - Voucher: Dupetit-Thouars s.n. (P?, n.v.).

Shrubs to small trees up to $9 \mathrm{~m}$ high, dbh up to $25 \mathrm{~cm}$, monoecious to dioecious. Indumentum composed of short and long simple hairs and sessile peltate-stellate hairs with a central cell. Resin yellowish. Stipules linear-triangular, $6.5-20$ by $0.5-0.8$ $\mathrm{mm}$, persistent to caducous, margin entire, apex acute, sparsely hairy on both sides. Leaves: petiole $22-134$ by $1-3 \mathrm{~mm}$, not grooved, sparsely hairy; blade ovate, $7-24$ by 4-12 cm, length/width ratio $2-2.5$, coriaceous, base not peltate, rounded to obtuse to acute, margin entire to crenate, apex acute to acuminate, upper surface glabrous, lower surface sparsely hairy on midrib and nerves, glabrescent to subglabrous, nerves 7-13 per side, looping close to the margin, with hair-tuft domatia. Inflorescences single to 2 together, unisexual or bisexual; peduncle 8-30 mm long; bracts linear-triangular, margin entire, apex acute, upper surface sparsely hairy to scattered, lower surface subglabrous. Flowers: pedicels sparsely hairy; sepals: margin entire, apex acute, sparsely hairy outside, subglabrous inside. Staminate inflorescences racemes, sometimes panicles, 3-10 cm long, 1-3 flowers per node, nodes per branch 5-30; bracts $2.5-7$ by 0.3-0.4 mm; bracteoles absent. Staminate flowers 5-6 mm diam.; pedicels 2.8-3.5 mm long; sepals 2 or 3, ovate to obovate, $3.5-4$ by $4-4.5 \mathrm{~mm}$, sparsely hairy outside, subglabrous inside; stamens 200-250, glabrous, filaments $0.5-0.8 \mathrm{~mm}$ long, thecae ovoid, $0.2-0.3$ by $0.2-0.25 \mathrm{~mm}$, connective not widened. Pistillate inflorescences racemes and/or developing one terminal flower, $8-10 \mathrm{~cm}$ long; bracts $5-10,6-10$ by $0.5-0.8 \mathrm{~mm}$, nodes per branch (when racemes) $3-5$, bracteoles $1-3$ by $0.2-0.25 \mathrm{~mm}$, early caducous. Pistillate flowers $2.8-3.5 \mathrm{~mm}$ diam.; pedicels $1-1.5 \mathrm{~mm}$ long; sepals 4 or 5 , linear-triangular, $5-7$ by $0.5-0.8 \mathrm{~mm}$, valvate, ovary $2-2.5$ by $2-2.5 \mathrm{~mm}$, densely hairy, 3-locular; style 0.5-2 mm long; stigma 10-25 mm long; staminodes sometimes present. Fruits $10-14$ by $12-20 \mathrm{~mm}$, outer surface subglabrous, spines 6 or 8 (see note), slightly curved, $3-10$ by $0.8-1.5 \mathrm{~mm}$, sparsely hairy; wall $0.8-1 \mathrm{~mm}$ thick; column $5-6$ by $5-6 \mathrm{~mm}$. Seeds $7-7.5$ by $5.5-6$ by $5.5-6 \mathrm{~mm}$, surface smooth, dull, brown.

Distribution - Mauritius and Réunion.

Habitat \& Ecology - Degraded wet forest and marshy ground. Altitude 200-700 m. Flowering and fruiting: November to May.

Note - Bernardi 14966 has bifurcating spines.

4. Cordemoya spinulosa (McPherson) S.E.C. Sierra, Kulju \& Welzen, comb. nov. Fig. 5c, d; Map 1

Mallotus spinulosus McPherson, Bull. Mus. Natl. Hist. Nat., B, Adansonia IV, 17 (1995) 170. Type: Schatz \& Lowry 1301 (holo MO; iso G, K, P, S, WAG, see note), Madagascar, Tamatave, Ambila-Lemaitso. 
Shrubs up to $4 \mathrm{~m}$ high, monoecious to dioecious. Indumentum composed of short simple and sessile peltate-stellate hairs with a central cell. Stipules linear-triangular, $2-5$ by 0.3-0.6 mm, early caducous, margin entire, apex acute, subglabrous on both sides. Leaves: petiole $13-55$ by $0.8-1.2 \mathrm{~mm}$, usually grooved, subglabrous; blade ovate to elliptic, $8-14$ by $3-6 \mathrm{~cm}$, length/width ratio $2.3-2.6$, coriaceous, base not peltate, obtuse to acute, margin entire to crenate, apex acuminate, upper and lower surface (sub)glabrous, nerves 6-9 per side, looping close to the margin, with hair-tuft domatia. Inflorescences single, unisexual to bisexual; bracts: margin entire, apex acuminate, hairy or not, upper surface sparsely hairy, lower surface glabrous; bracteoles absent. Flowers: pedicels glabrous; sepals: margin entire, apex acute, subglabrous to glabrous outside, glabrous inside. Staminate inflorescences racemes, 2-5 cm long, peduncle 3-4 mm long, 1-3 flowers per node, nodes per branch 7-23; bracts triangular, 0.5-0.8 by $0.3-0.4 \mathrm{~mm}$; bracteoles absent. Staminate flowers $4.5-5 \mathrm{~mm}$ diam.; pedicels 3-4 $\mathrm{mm}$ long; sepals 3 , ovate to obovate, $1.8-2.2$ by $1.8-2 \mathrm{~mm}$, glabrous; stamens $70-90$, glabrous, filaments $1-2 \mathrm{~mm}$ long, red, thecae ovoid, $0.2-0.3$ by $0.2-0.25 \mathrm{~mm}$, brownish red, connective not widened. Pistillate inflorescences racemes or developing one terminal flower, $1.5-3 \mathrm{~cm}$ long; bracts $4-8$, narrowly triangular, $1.2-2$ by $0.2-0.4$ $\mathrm{mm}$, nodes (when racemes) 4; bracteoles absent. Pistillate flowers $1.8-2 \mathrm{~mm}$ diam.; pedicels $0.8-1 \mathrm{~mm}$ long; sepals 5 or $6,1.5-2.2$ by $0.2-0.3 \mathrm{~mm}$, valvate, sparsely hairy outside, subglabrous inside; staminodes absent; ovary $1.5-1.7$ by $1.5-1.7 \mathrm{~mm}$ (covered with a resin), 2- or 3-locular; style $0.5-1 \mathrm{~mm}$ long; stigma 5-10 mm long. Fruits 5-6 by $8-9 \mathrm{~mm}$, green, outer surface subglabrous, spines 4 or 6 , slightly curved, $2-4$ by $0.8-1 \mathrm{~mm}$, subglabrous; wall $0.3-0.5 \mathrm{~mm}$ thick; column $3.5-4$ by c. $3 \mathrm{~mm}$. Seeds $4-4.2$ by $4-4.1$ by $4-4.1 \mathrm{~mm}$, surface smooth, shiny, dark brown.

Distribution - Endemic to Madagascar.

Habitat \& Ecology - Coastal dune forest, sclerophyllous mountain forest. Altitude: sea level up to $1240 \mathrm{~m}$. Flowering and fruiting: August to May.

Vernacular names - Madagascar: Ampalay rano.

Note - McPherson (1995) lists the following additional isotypes: BR, C, DAV, DSM, EA, ERE, GH, IT, LE, NY, PRE, SRGH, TAN, US.

\section{Subgenus Diplochlamys}

Subgenus Diplochlamys (Müll.Arg.) S.E.C. Sierra, Kulju \& Welzen, comb. nov.

Diplochlamys Müll.Arg., Flora 47 (1864) 539; Müll.Arg. (1866) 1023. - Mallotus Lour. sect. Diplochlamys (Müll. Arg.) Baill. ex T. Durand (1888) 370; Müll.Arg. ex Pax (1890) 55, isonym. -Type: Diplochlamys griffithianus Müll.Arg. [= Cordemoya griffithiana (Müll.Arg.) S.E.C. Sierra, Kulju \& Welzen].

Hancea Seem. (1857) 409. - Mallotus Lour. sect. Hancea (Seem.) Pax \& K. Hoffm. (1914) 199. - Type: Hancea hookeriana Seem. [= Cordemoya hookeriana (Seem.) S.E.C. Sierra, Kulju \& Welzen].

Mallotus Lour. sect. Oliganthae Airy Shaw 21 (1968) 389. - Type: Mallotus subpeltatus Blume [= Cordemoya subpeltata (Blume) M. Aparicio].

Branches with slender or conspicuously swollen nodes. Indumentum composed of simple hairs and capitate glandular hairs with multicellular stalks. Stipules interpetiolar 
or axillary, margin entire or serrate. Leaves opposite (strongly unequal) or alternate; blade peltate or not, coriaceous to chartaceous, margin entire (rarely serrate), extrafloral nectaries absent, venation pinnate or triplinerved (rarely palminerved), looping close to the margin (rarely ending in the margin). Inflorescences axillary or ramiflorous, with one flower per bract. Staminate inflorescences racemes or panicles. Male flowers: sepals ( 2 or) 3 or 4 , free to basally connate; connective sometimes widened. Pistillate inflorescences racemes and/or developing one terminal flower. Pistillate flowers: 3- (or 4-)locular. Fruits opening loculicidally-septicidally or septicidally-loculicidally, inside glabrous to densely hairy.

Distribution - From Burma to South China throughout Southeast Asia and Malesia to New Guinea.

\section{Section Diplochlamys}

Section Diplochlamys (Müll.Arg.) S.E.C. Sierra, Kulju \& Welzen, comb. nov.

Diplochlamys Müll.Arg., Flora 47 (1864) 539; Müll.Arg. (1866) 1023. - Mallotus Lour. sect. Diplochlamys (Müll.Arg.) Baill. ex T. Durand (1888) 370; Müll.Arg. ex Pax (1890) 55, isonym. - Type: Diplochlamys griffithianus Müll.Arg. [= Cordemoya griffithiana (Müll.Arg.) S.E.C. Sierra, Kulju \& Welzen].

Hancea Seem. (1857) 409. - Mallotus Lour. sect. Hancea (Seem.) Pax \& K. Hoffm. (1914) 199.

- Type: Hancea hookeriana Seem. [= Cordemoya hookeriana (Seem.) S.E.C. Sierra, Kulju \& Welzen].

Branches with conspicuously swollen nodes. Stipules interpetiolar, margin entire. Leaves opposite, strongly unequal, one of each pair stipuliform; blade not peltate, margin entire (rarely serrate), venation pinnate, rarely palminerved, looping close to the margin, rarely ending in the margin. Inflorescences axillary. Staminate inflorescences racemes or panicles. Staminate flowers: sepals ( 2 or) 3 or 4 , free to basally connate. Pistillate inflorescences racemes and/or developing one flower terminally. Pistillate flowers: sepals (3 or) 4-6; ovary 3- (or 4-)locular. Fruits opening septicidally-loculicidally, inside glabrous to hairy, spines with or without terminal glands.

Distribution - From South China throughout Southeast Asia and Malesia to New Guinea.

Descriptions, typification, synonymy, distribution maps, illustrations, and further details of the species of former Mallotus sect. Hancea can be found in Slik \& Van Welzen (2001a). The type material mentioned on the list below was not studied during the present revision.

New combinations in Cordemoya sect. Diplochlamys (Müll.Arg.) S.E.C. Sierra, Kulju \& Welzen:

5. Cordemoya cordatifolia (Slik) S.E.C. Sierra, Kulju \& Welzen, comb. nov.

Mallotus cordatifolius Slik, Blumea 43 (1998) 225. - Type: PNH (Gutierrez et al.) 117545 (holo

L), Philippines, Samar Island, Mt Sohoon. 
6. Cordemoya eucausta (Airy Shaw) S.E.C. Sierra, Kulju \& Welzen, comb. nov.

Mallotus eucaustus Airy Shaw, Kew Bull. 23 (1969) 80. - Type: S (Sibat ak Luang) 23630 (holo $\mathrm{K}$; iso L), Borneo, Sarawak, Third Division, Bukit Iju, Ulu Arip, Balingian.

7. Cordemoya grandistipularis (Slik) S.E.C. Sierra, Kulju \& Welzen, comb. nov.

Mallotus grandistipularis Slik, Blumea 43 (1998) 227. - Type: Burley, Tukirin et al. 2021 (holo L; iso CANB, K, US), Indonesia, Sumatra, Riau Province, Tigapulu, $5 \mathrm{~km} \mathrm{~W}$ of Talanglakat.

8. Cordemoya griffithiana (Müll.Arg.) S.E.C. Sierra, Kulju \& Welzen, comb. nov. Diplochlamys griffithianus Müll.Arg., Flora 47 (1864) 539. - Type: Griffith KD 4961 (holo K).

9. Cordemoya hirsuta (Elmer) S.E.C. Sierra, Kulju \& Welzen, comb. nov.

Mallotus hirsutus Elmer, Leafl. Philipp. Bot. 7 (1915) 2648. - Type: Elmer 13480 (holo PNH†; iso K, L, NY), Philippines, Mindanao, Agusan Province, Cabadbaran (Mt Urdaneta).

10. Cordemoya hookeriana (Seem.) S.E.C. Sierra, Kulju \& Welzen, comb. nov.

Hancea hookeriana Seem., Bot. Voy. Herald (1857) 409. - Type: Champion s.n. (holo K), Hong Kong.

11. Cordemoya kingii (Hook.f.) S.E.C. Sierra, Kulju \& Welzen, comb. nov.

Mallotus kingii Hook.f., Fl. Brit. India 5 (1887) 439. - Type: King's collector 7414 (holo K; iso G, SING), Malaya, Peak.

12. Cordemoya longistyla (Merr.) S.E.C. Sierra, Kulju \& Welzen, comb. nov.

Mallotus longistylus Merr., Philipp. J. Sci. 16 (1920) 560. — Type: BS (Ramos \& Pascasio) 35173 (holo PNH†; iso K), Philippines.

13. Cordemoya papuana (J.J. Sm.) S.E.C. Sierra, Kulju \& Welzen, comb. nov.

Mallotus hookerianus (Seem.) Müll.Arg. var. papuanus J.J. Sm., Nova Guinea 8, 4 (1912) 787, t. 137. - Type: Gjellerup 316 (holo L; iso A, BO, K, P, U), Irian Jaya, Bivak Hollandia.

14. Cordemoya penangensis (Müll.Arg.) S.E.C. Sierra, Kulju \& Welzen, comb. nov.

Mallotus penangensis Müll.Arg., Linnaea 34 (1865) 186. - Type: Wallich 8576 (holo G; iso A, K, P), Malaysia, Pulau Penang.

15. Cordemoya stipularis (Meijer ex Airy Shaw) S.E.C. Sierra, Kulju \& Welzen, comb. nov.

Mallotus stipularis Meijer [Bot. News Bull. Forest Dept., Sabah 7 (1967) 53, anglice] ex Airy Shaw, Kew Bull. 21 (1968) 398. - Type: Lörzing 14746 (holo K; iso L), Sumatra, N Sibajak, W of Bandarbaru.

16. Cordemoya wenzeliana (Slik) S.E.C. Sierra, Kulju \& Welzen, comb. nov.

Mallotus wenzelianus Slik, Blumea 43 (1998) 229. - Type: Wenzel 2697 (holo G; iso A, UC), Philippines, Mindanao, Surigao Province, Jubud. 


\section{Section Oliganthae}

Section Oliganthae (Airy Shaw) S.E.C. Sierra, Kulju \& Welzen, comb. nov.

Mallotus Lour. sect. Oliganthae Airy Shaw, Kew Bull. 21 (1968) 389. - Type: Mallotus subpeltatus Blume [= Cordemoya subpeltata (Blume) M. Aparicio].

Branches with slender nodes. Stipules axillary, margin serrate. Leaves alternate; blade peltate, margin entire, venation triplinerved, looping close to the margin. Inflorescences ramiflorous to axillary. Staminate inflorescences racemes. Staminate flowers: sepals 3 (or 4), basally connate. Pistillate inflorescences developing one terminal flower. Pistillate flowers: sepals 5-7; ovary 3-locular. Fruits opening loculicidally-septicidally, inside densely hairy, spines without terminal glands.

Distribution - From Burma to Thailand, Peninsular Malaysia, Sumatra, Borneo and Java.

17. Cordemoya subpeltata (Blume) M. Aparicio, comb. nov. - Fig. 6; Map 2

Adisca subpeltata Blume, Bijdr. 11 (1826) 610. - Rottlera subpeltata (Blume) Baill. (1858) 423.

- Mallotus subpeltatus (Blume) Müll.Arg. (1865) 189. - Lectotype (selected here): Blume

s.n. '1510' (holo L), Java, Krawong.

Mappa rhynchophylla Miq. (1859) 403. - Rottlera rhynchophylla (Miq.) Miq. (1861) 181, 454.

- Lectotype (selected here): Teijsmann HB 3645 (lecto U (see note)), Sumatra, Tubuan, Ogan Ulu.

Mappa acuminatissima Zipp. ex Span. (1841) 349, nom. nud. - Voucher: Herb. Zippelius (L?).

Shrubs to small trees up to $15 \mathrm{~m}$ high, dbh up to $15 \mathrm{~cm}$, dioecious. Outer bark smooth, grey-brown. Indumentum composed of short (with a pointed tip), long simple and tufted hairs, and capitate glandular hairs with multicellular stalks ${ }^{1}$. Stipules narrowly triangular, $5-7$ by $1.8-2 \mathrm{~mm}$, caducous to early caducous, margin serrate, hairy, apex acute, sparsely to densely hairy on both sides. Leaves: petiole $1-14 \mathrm{~cm}$ by $1-1.3 \mathrm{~mm}$, not grooved, glabrescent; blade elliptic to narrowly ovate, $7.5-27$ by $6-11 \mathrm{~cm}$, length/ width ratio $2.5-3$, chartaceous, base subpeltate up to $2 \mathrm{~mm}$, rounded to obtuse, margin entire, apex caudate, upper surface sparsely hairy, glabrescent, lower surface sparsely hairy on midrib and nerves, with few glandular hairs, nerves 5-7 per side, looping close to the margin, with hair-tuft domatia. Inflorescences ramiflorous or axillary, single or 2 together, unisexual; peduncle $0.5-1.2$ by c. $1 \mathrm{~mm}$; bracts triangular, $1.8-2.5$ by $1-1.5$ $\mathrm{mm}$, persistent, margin entire, apex acute, upper surface hairy, lower surface densely hairy; bracteoles absent. Flowers: pedicels densely hairy; sepals margin entire, apex acute. Staminate inflorescences racemes, $0.5-0.8 \mathrm{~cm}$ long, nodes per branch 3-6; bracteoles absent. Staminate flowers 5-7 mm diam.; pedicels 5-7 mm long; sepals 3 (or 4 ), ovate, $4.5-5$ by $2-2.5 \mathrm{~mm}$, densely hairy outside, subglabrous to sparsely hairy inside; stamens 200-250, glabrous, filaments 2-7 mm long, thecae ellipsoid, 0.5-0.7 by $0.4-0.5 \mathrm{~mm}$, connective widened. Pistillate inflorescences developing one terminal flower, 1.5-3 cm long; bracts 4 or 5; bracteoles absent. Pistillate flowers c. $5 \mathrm{~mm}$ diam.;

1) The presence of glandular hairs in C. subpeltata (formerly M. subpeltatus) has been ambiguous. Backer \& Bakhuizen van den Brink f. (1964) observed their presence, while Airy Shaw (1968, 1974); Welzen, Slik \& Bollendorff in Van Welzen et al. (2000), noted their absence. However, recent leaf anatomical studies by Ž. Fišer (in prep.) have confirmed their presence (Fig. 3c). 


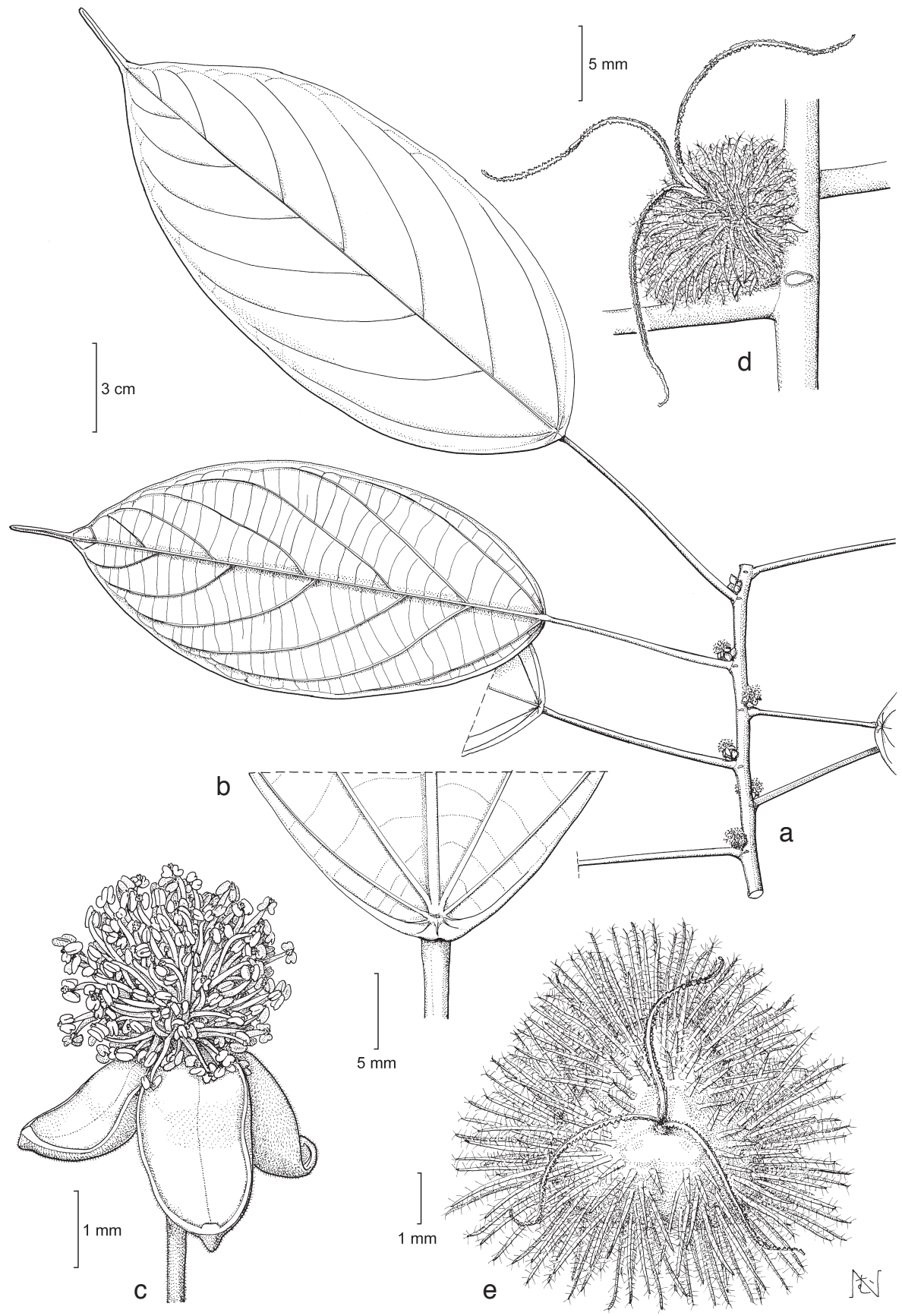

Fig. 6. Cordemoya subpeltata (Blume) M. Aparicio. a. Habit with male flowers; b. base of leaf upper surface; c. staminate flower; d. young fruit; e. mature fruit (a-c: Van Beusekom \& Phengkhlai 533, L; d: Prawiroatmodjo 361, BO; e: Maxwell 86-514, A). 


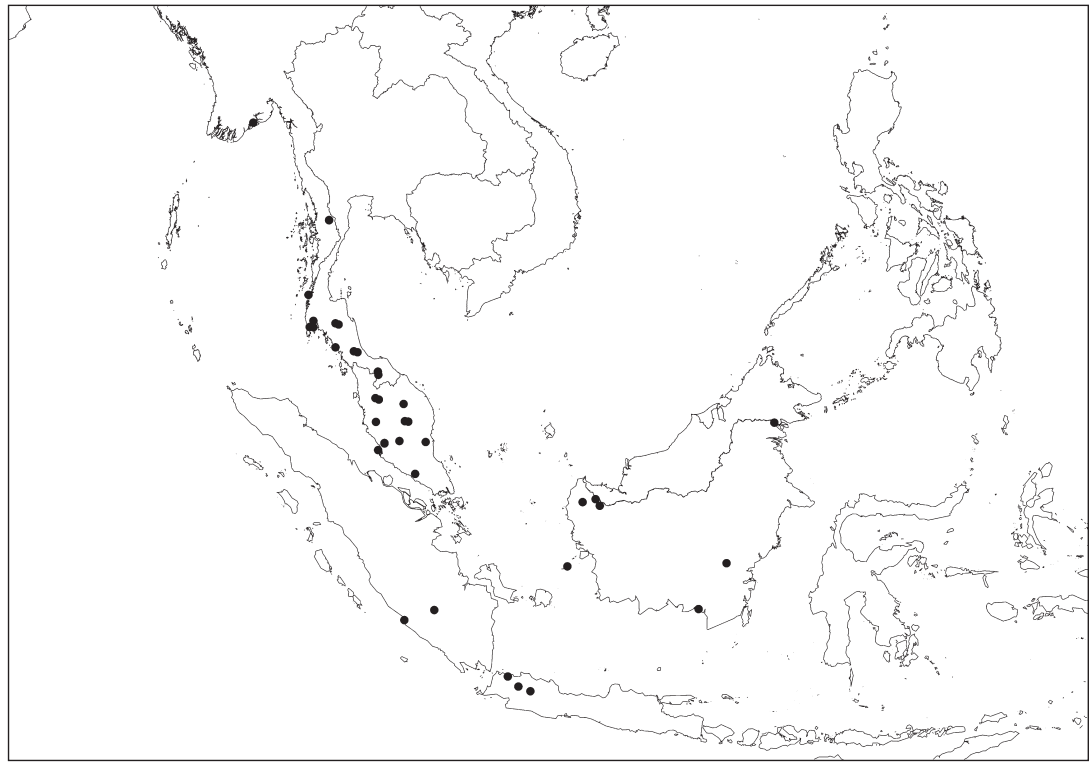

Map 2. Distribution of C. subpeltata (Blume) M. Aparicio.

pedicels $0.7-2 \mathrm{~mm}$ long; sepals 5 or 6 , narrowly triangular, $4-6$ by $1-1.5 \mathrm{~mm}$, valvate and/or imbricate, hairy outside and inside; staminodes absent; ovary $1-2$ by $2-3 \mathrm{~mm}$, 3-locular, densely hairy; style $1-1.5 \mathrm{~mm}$ long; stigma $8-12 \mathrm{~mm}$ long. Fruits $8-15$ by $15-20 \mathrm{~mm}$, pale green, outer surface densely hairy, spines $140-170$, straight, $6-10$ by $1 \mathrm{~mm}$, densely hairy; wall 1-2 mm thick, inside densely hairy; column $9.5-15$ by $9-14$ $\mathrm{mm}$. Seeds $9-12$ by $9-11$ by $8-10 \mathrm{~mm}$, surface smooth, shiny, dark brown.

Distribution - From Burma to Thailand, Peninsular Malaysia, Sumatra, Borneo and Java.

Habitat \& Ecology - Locally in understory of evergreen forest. Altitude: sea level up to 1400 m. Flowering: February to May; fruiting: June to January.

Note - Miquel in 1861 mentions that the Zollinger collection cited in 1859 comes from Sumatra and not from Java. Additionally he mentions two collections by Teijsmann in Sumatra. We did not find any collections with the name Mappa rhyncophylla Miq. from Zollinger in Sumatra, therefore, we have appointed as lectotype one of the collections from Teijsmann in Sumatra.

\section{ACKNOWLEDGEMENTS}

The directors of the following institutes are thanked for loans of their material: A, BM, BO, G, K, L, MO, P, S, U, WAG. Special thanks are due to J.F. Veldkamp for his valuable help concerning nomenclatural problems; P. Baas for his comments on leaf anatomy; M. Nakajima and A. Walsmit Sachs for the drawings; N. Sol, B. Vrijbloed and G. Thijsse for the administration of the collections, L. Frankova and D.N.F. Kiehl for help with the literature; J.M. de Wolf, B.J. van Heuven for technical support; J.B. Mols and J.W.F. Slik for their comments on the manuscript. 


\section{REFERENCES}

Airy Shaw, H.K. 1968. Malesian and other Asiatic Euphorbiaceae. Kew Bull. 21: 379-400.

Airy Shaw, H.K. 1969. Malesian and other Asiatic Euphorbiaceae. Kew Bull. 23: 80, 81.

Airy Shaw, H.K. 1974. Noteworthy Euphorbiaceae from tropical Asia. Hooker's Icon. Pl. 38, 1: $1-3$, f. 1 .

Backer, C.A. \& R.C. Bakhuizen van den Brink Jr. 1964. Flora of Java 1. Noordhoff, Groningen.

Baillon, H.E. 1858. Étude générale du groupe des Euphorbiacées. Masson, Paris.

Baillon, H.E. 1861. Species Euphorbiacearum. Euphorbiacées africaines. Deuxième partie, Afrique orientale (Bourbon, Maurice, Madagascar, Zanguebar, etc.). Adansonia 1: 255.

Baillon, H.E. 1891a. Liste des plantes de Madagascar. Bull. Mens. Soc. Linn. Paris 2: 977-978.

Baillon, H.E. 1891b. In: A. Grandidier, Histoire physique, naturelle et politique de Madagascar: Histoire naturelle des plantes 34: t. 175. Grandidier, Paris.

Bentham, G. 1880. Euphorbiaceae. In: G. Bentham \& J.D. Hooker, Genera Plantarum 3: 319-320. Reeve \& Co, London.

Blume, C.L. 1826. Bijdragen tot de Flora van Nederlandsch-Indië 11: 610. Lands drukkerij, Batavia.

Bojer, W. 1837. Hortus Mauritianus. Aime Mararot \& Co., Mauritius.

Bojer, W. 1846. In: L. Bouton, Trav. Soc. Hist. Nat. Maurice 1842-1846: 151-153.

Coode, M.J.E. 1982. Euphorbiaceae. In: Flora des Mascareignes 160: 50-51. The Sugar Industries Research Institute, Mauritius. L'office de la Recherche Scientifique et Technique outre-mer, Paris; The Royal Botanical Gardens, Kew.

De Candolle, A.P. 1838. Revue sommaire de la famille des Bignoniacées. Biblioth. Universelle Genève II, 17: 134, reprinted in Ann. Sci. Nat., Bot. II, 11 (1839): 296.

Durand, T.A. 1888. Index generum phanerogamorum: 370 . Durand, Brussel, etc.

Elmer, A.D.E. 1915. Two hundred twenty six new species. Leafl. Philipp. Bot. 7: 2648.

Forster, G. 1786. Florulae insularum Australium prodromus. Joann, Christian \& Dieterich, Gottingae. Hooker, J.D. 1887. Flora of British India 5. Reeve \& Co., Brook nr Ashford.

Léandri, J. 1957 ('1956’). Euphorbiacee malgaches nouvelles recoltées par M.R Capuron. Bull. Soc. Bot. France 103: 605-606.

McPherson, G. 1995. On Mallotus and Deuteromallotus (Euphorbiaceae) in Madagascar. Bull. Mus. Natl. Hist. Nat., B, Adansonia IV, 17: 170.

Meijer, W. 1967. Euphorbiaceae. Key to about 50 arboreous genera occurring in Sabah. Bot. News Bull. Forest Dept., Sabah 7: 53.

Merrill, E.D. 1920. Notes on Philippine Euphorbiaceae, III. Philipp. J. Sci. 16: 559-561.

Miquel, F.A.W. 1859. Flora van Nederlandsch Indië. Van der Post, Amsterdam.

Miquel, F.A.W. 1861. Flora van Nederlandsch Indië, Eerste Bijvoegsel. Van der Post, Amsterdam.

Müller Argoviensis, J. 1864. Neue Euphorbiaceen des Herbarium Hooker in Kew. Flora 47: 539.

Müller Argoviensis, J. 1865. Euphorbiaceae. Linnaea 34: 184-197.

Müller Argoviensis, J. 1866. Euphorbiaceae tribus Acalypheae. In: A. de Candolle, Prodromus Systematis Naturalis Regni Vegetabilis 15, 2: 956-983, 1023. Masson \& Fili, Paris.

Pax, F.A. 1890. Euphorbiaceae. In: A. Engler, Die natürlichen Pflanzenfamilien 3, 5: 53-55. Engelmann, Leipzig.

Pax, F.A. \& K. Hoffmann. 1914. Euphorbiaceae-Acalypheae-Mercurialinae. In:A. Engler, Das Pflanzenreich IV.147.vii: 145-212, 396-397. Engelmann, Leipzig.

Roxburgh, W. 1832. Flora Indica 3. Thacker \& Co., Calcutta.

Seemann, B.C. 1857. Flora of the Island of Hong Kong. The Botany of the Voyage of H.M.S. Herald: 409-410. Reeve, London.

Slik, J.W.F. 1998. Three new Malesian species of Mallotus section Hancea (Euphorbiaceae). Blumea 43: $225-232$.

Slik, J.W.F. \& P.C. van Welzen. 2001a. A taxonomic revision of Mallotus sections Hancea and Stylanthus (Euphorbiaceae). Blumea 46: 3-66.

Slik, J.W.F. \& P.C. van Welzen. 2001b. A phylogeny of Mallotus (Euphorbiaceae) based on morphology: Indications for a pioneer origin of Macaranga. Syst. Bot. 26: 786-796.

Smith, J.J. 1912. Euphorbiaceae. Nova Guinea 8, 4: 779-796, t. 130-142. 
Spanoghe, J.B. 1841. Prodromus Florae Timorensis. Linnaea 15: 349.

Steudel, E.T. 1841. Nomenclatur Botanicus 2: 459. J.G. Cottae, Stutgartiae \& Tubingae.

Van Welzen, P.C., R.M.A.P. Haegens, J.W.F. Slik, S.M. Bollendorff, S. Dressler \& H.-J. Esser. 2000.

Checklist of the genera of Thai Euphorbiaceae. Thai Forest Bull., Bot. 28: 93-111.

Webster, G.L. 1994. Synopsis of the genera and suprageneric taxa of Euphorbiaceae. Ann. Missouri Bot. Gard. 81: 33-144.

Willdenow, C.L. 1805. Species Plantarum, ed. 4, 1. Nauk, Berlin.

\section{IDENTIFICATION LIST FOR SECTION CORDEMOYA AND OLIGANTHAE}

(for Sect. Diplochlamys see Slik, 2001a)

The numbers behind the collector numbers refer to the following taxa:

$$
\begin{aligned}
1 & =\text { C. acuminata (Baill.) Baill. } \\
2 & =\text { C. capuronii }(\text { Léandri) S.E.C. Sierra, Kulju \& Welzen } \\
3 & =\text { C. integrifolia } \text { (Willd.) Baill. } \\
4 & =\text { C. spinulosa (McPherson) S.E.C. Sierra, Kulju \& Welzen } \\
17 & =\text { C. subpeltata (Blume) M. Aparicio }
\end{aligned}
$$

Abu 3334: 17 - Alvins 2097: 17 - Andrianjafy et al. 168: 2 - Aridy \& Moïse 184: 1 - Aridy \& Rahajasoa 96: 1.

Bakhuizen van den Brink Jr. 2485: 17 - bb series 35757: 17 - Bernardi 14782: 3; 14966: 3 Beumée A153: 17; 5671: 17; 9316: 17 - J. Blackburn 106: 3 - Blume s.n. '1510': 17 - Bosser 11728: 3 - Burley et al. 2925: 17.

Cadet 564: 3 - Coode \& Cadet 4958: 3 - Coode, Gueho \& Lorence 3952: 3; $4754: 3$ - C. Curtis 1557: 17; 2376: 17 .

d'Alleizette 6522b:1 - B. Danser 5469: 17 - Decary 16684: 1.

Frappier 278: 3.

Gautier \& Chetelain 2690: 4 - Gautier, Messmer \& Andriatsiferana 3547: 2; 3615: 2 - Gautier \& Totozafy Be 2910: 4 - R. Geesink 6420: 17; 7245: 17 - R. Geesink \& Santisuk 5426: 17 - Goodenough 16529: 17 - Griffith KD 4759: 17.

Hardial \& Samsuri 194: 17 - Herb. Willdenow 17934: 3.

Kadim 96: 17 - KEP series 99150: 17; 104393: 17 - KEP FRI series 3136: 17; 3354: 17; 3439: 17; 4351: 17; 8507: 17; 8602: 17; 13281: 17; 14421: 17; 16096: 17; 18403: 17; 20975: 17; 39484: 17 - Kerr 16352: 17; 18659: 17 - Kiah 23999: 17; 24324: 17 - King's collector 5844: 17 — KL series 2704: 17; 3059: 17 - Koorders 24058: 17; 30974: 17 - Kostermans 12001: 17 _ A. Kotozafy 999: 2 - Koyama et al. 34080: 17.

Labat \& Andrianjafy 3399: 2 - Lam \& Meeuse 5970: 1 - Laman 1036: 17 - Laman, Rachman \& Mirmato 1280: 17 - K. Larsen et al. 45424: 17 - Lorence 2307: 3.

Maxwell 75-274: 17; 75-891: 17; 84-102: 17; 86-77: 5; 86-133: 17; 86-333: 5; 86-514: 17; 87-478: 17 - Messmer \& Andriatsiferana 653: 2; 698: 2 - Middleton 494: 17 - Middleton et al. 1735: 17 - Mocquerys 244: 1.

Newman 1029: 17 - Newman et al. 1071: 17.

Pinnin et al. 436: 17 - Prawiroatmodjo 361: 17.

Rabevohitra 2052: 4 - Rakotomalala \& Narison 171: 2 - Rakotomalaza, Messmer \& Rakotovao 1502: 2; 1502A: 2 - Rakotomalaza, Messmer \& Ravelonarivo 730: 1 - Randriamampionona 670: 1 - Randrianasolo \& Bernardin 231: 1 - Ratovoson et al. 573:2 - Ravelonarivo \& Rabesonina 653: 2 - Reksodihardjo 658: 17 - Ridley 2298: 17; 8187: 17; 15792: 17; 58525: 17.

SAN series 89785: 17 - Schatz \& Lowry 1301: 4 - Scortechini 1723: 17 - SF series 16885: 17; 22715: 17; 23999: 17; 24385: 17; 40575: 17 - SF (Mad) series 8713: 2; 9098: 2 - Shah 618: 17; 1500: 17 - Shah \& Noor 1942: 17 - Shah \& Shukor 2636: 17 - Soejarto 5948: 17 - Strugnell 13935: 17 - Suzuki 5235: 17.

Teijsmann HB 3052: 17; HB 3645: 17; HB 4266: 17 - S. Thaworn 583:5 - Thorenaar 489: 17; A 638: 17 - Totozafy Be 520: 4 . 
Van Beusekom \& Phengkhlai 533: 17 - Van Steenis 8847: 17; 12617: 17.

Wallich 7755: 17 - Wiriadinata 1075: 17 - Wray 3560: 17.

Yamada 9504: 17.

Zollinger 1001: 17.

\section{INDEX TO NAMES}

The numbers behind the names refer to the species number in this article. Accepted names are in roman type; synonyms in italics and new combinations in bold.

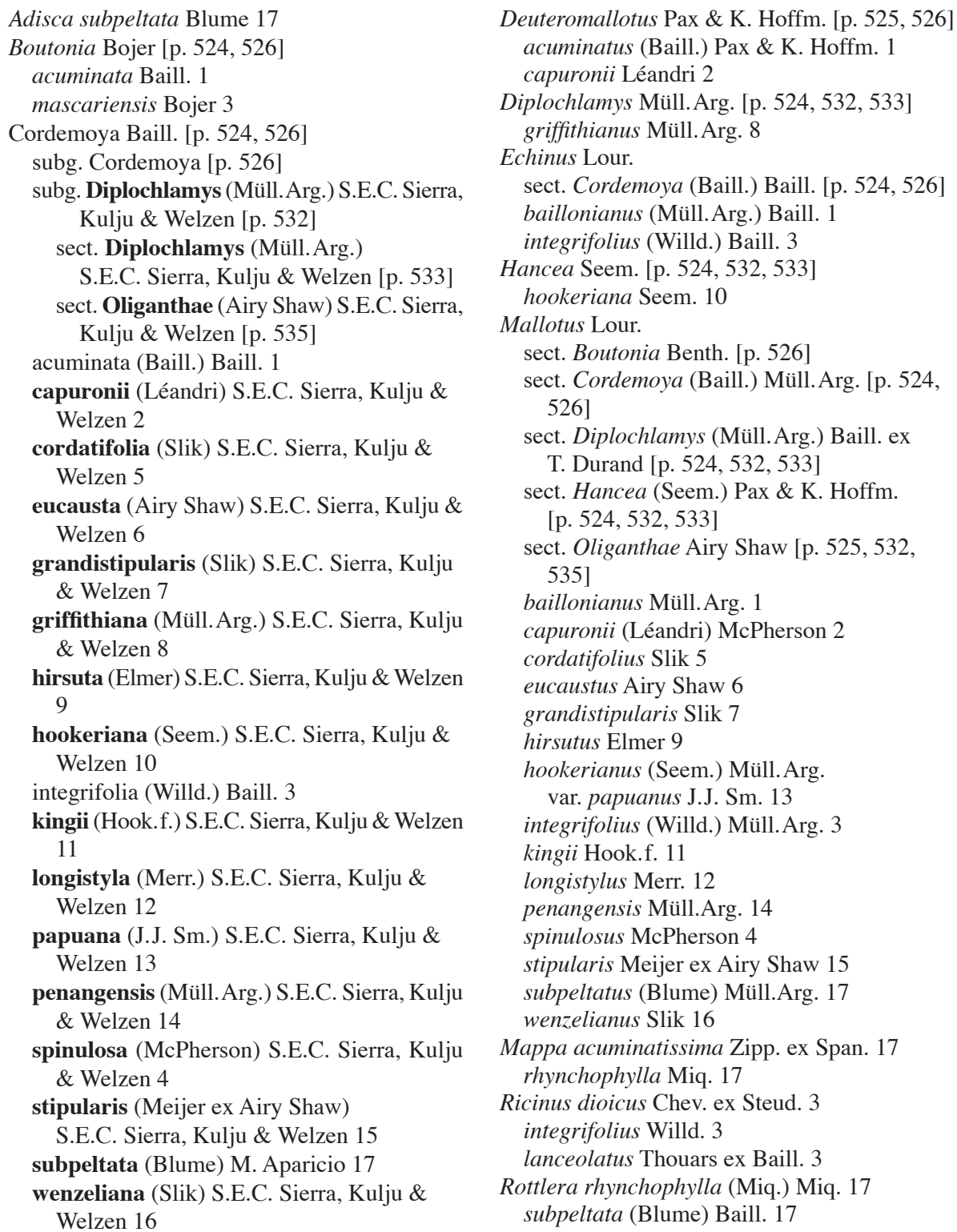

